Reprod. Nutr. Dévelop., 1988, 28 (1), 177-178.

\title{
Mise en évidence et signification de l'hétérogénéité contractile des parois du rumen
}

\author{
Y. RUCKEBUSCH, R. BAUMONT, C. H. MALBERT \\ Ecole Nationale Vétérinaire, 23, chemin des Capelles, \\ 31076 Toulouse Cedex, France.
}

Summary. The origin of pressure variations in the dorsal sac of the rumen, as a result of phasic contractions superimposed on changes in basal smooth muscle tone, was identified by means of curved strain-gauge force transducers fixed on the wall of different parts of the rumen. Both phasic and tonic activities of the wall of the ruminal ventral sac on its visceral (right) side were found to be the major determinant of these changes.

Si le degré de remplissage du réticulo-rumen peut être considéré globalement comme un facteur limitant l'ingestion de fourrages (Balch et Campling, 1962 ; Forbes, 1980), son contenu n'est pas réparti de façon homogène et l'extensibilité de ses parois n'est pas uniforme. Maintenu par les muscles de l'abdomen fixés au rachis, le rumen occupe la partie gauche de la cavité abdominale, de sorte que la distension est surtout celle des parois droites au détriment de la masse intestinale. C'est probablement en raison de l'absence de toute contrepression directe sur le sac ventral droit (SVD) que l'on enregistre à ce niveau chez la vache des variations négatives de pression (Ruckebusch et Kay, 1971). Dans cette étude, nous avons tenté de mesurer l'importance relative des déformations, actives et passives, des parois dorsales et ventrales du rumen chez la brebis debout ou couchée et dont le contenu intraruminal est normal ou liquide.

Matériel et méthodes. Chez des brebis adultes, munies d'une canule du sac dorsal gauche (SDG) du rumen, des jauges de contrainte légèrement incurvées $\left(1 \mathrm{~cm}^{2}\right)$ sont fixées sur la séreuse du rumen, à mi-distance entre les piliers séparant le SD et le SV et le bord supérieur ou inférieur du rumen. On enregistre par rapport aux variations de la pression intraruminale, les déformations correspondant aux contractions phasiques et toniques de la paroi, le tonus pariétal étant accru puis réduit (20-30 $\mathrm{min})$ par l'injection de pilocarpine puis d'atropine (50 $\mu \mathrm{g} . \mathrm{kg}$ ). L'activité alimentaire (foin de luzerne) et la position de l'animal sont identifiées ainsi que le début du cycle de contraction réticulo-ruminal (électromyographie). La déformation pleine échelle de l'ordre de $5 \mathrm{~g}$ des jauges est comparée chez la brebis couchée puis debout et dont le contenu du rumen est du foin ou un liquide (Malbert et Ruckebusch, 1987).

Résultats et discussion. Chez l'animal en décubitus latéral droit, la paroi du SDG (fig. 1 A) ainsi que la paroi du SVD (fig. 1 B) sont celles dont l'amplitude des déformations est maximale au cours d'une contraction primaire $(P)$. Chez l'animal debout, la paroi du SVD se relâche de façon importante comme durant les premières minutes de l'ingestion. Cette dernière réponse équivaut à la relaxation réceptive du fundus décrite initialement chez le chat par Jansson (1969) et retrouvée chez les autres espèces monogastriques. La contraction secondaire (S) 
du rumen correspond à une déformation maximale simple ou double de la paroi du SVD (fig. 1 A) ou de l'ensemble du SV (fig. 1 B), le relâchement postcontractile pouvant s'accompagner d'une diminution de la tension pariétale du sac dorsal. A noter que les contractions du SD (1 et 2) et du SV (3 et 4$)$ sont quasi instantanées après liquéfaction du contenu ruminal. Enfin, les variations provoquées de tonus sont toujours importantes pour l'énorme SVD, tel qu'il a été visualisé par tomographie (Reid, 1986).

En résumé, I'hétérogénéité contractile des parois du rumen est maximale pour la partie droite du sac ventral, dont la déformation n'est pas limitée par la sangle abdominale.

FIG. 1. - Mécanogrammes

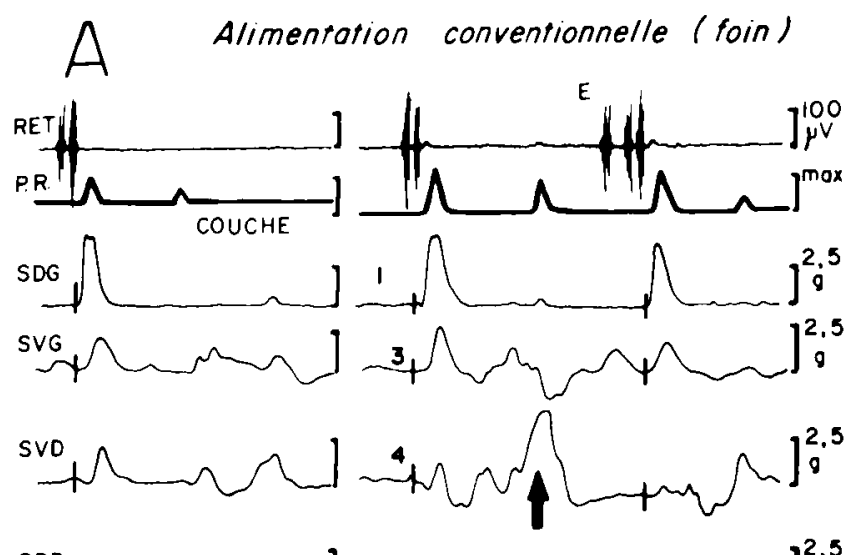
correspondant à la déformation active des parois du sac dorsal gauche (SDG) et droit (SDD) du rumen et du sac ventral gauche (SVG) et droit (SVD) à la suite des contractions biphasiques ou avec extracontraction (E) du réseau (RET) chez la même brebis en décubitus latéral droit ou debout, dont le contenu ruminal est normal (A) ou liquide (B). Les variations de la pression résultante (PR) correspondent pour les contractions secondaires à celles du SVD en $A$ ou de l'ensemble du $S V$ lorsque le contenu du rumen est liquide (flèche).
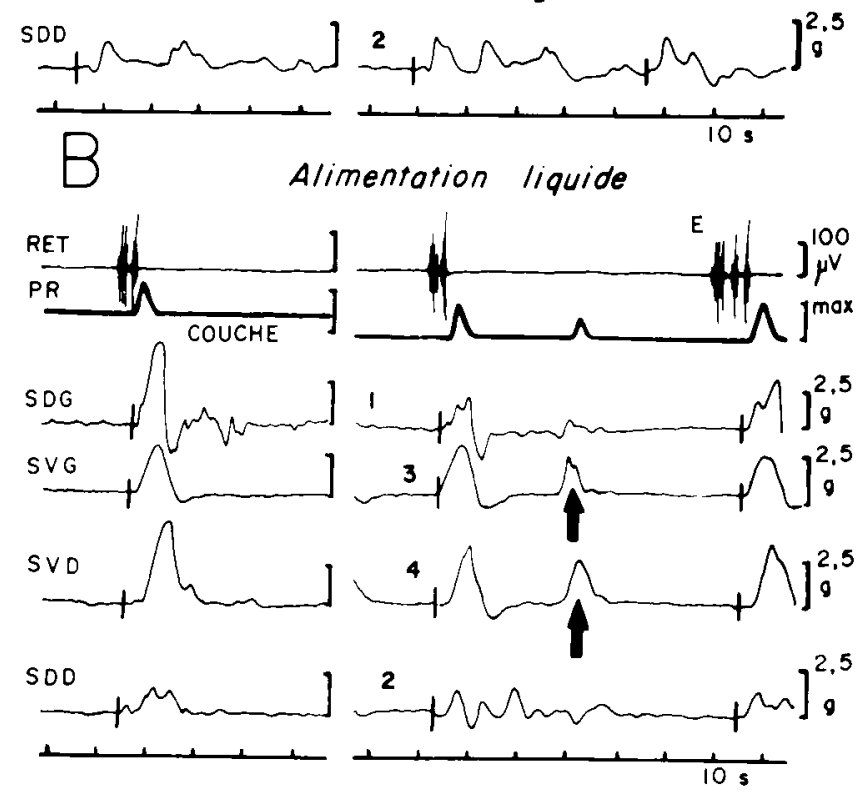

Balch C. C., Campling R. C., 1962, Nutr. Abstr. Rev., 32, 669-686.

Forbes J. M., 1980. Appetite, 1, 21-41.

Jansson G., 1969. Acta physiol. scand., 326, 1-42.

Malbert C. H., Ruckebusch Y., 1987. Revue Méd. vét., 138, 155-161.

Reid C. S. W., 1986. In : Control of digestion and metabolism in ruminants, L. P. Milligan et al. (eds),

A. Reston Book, Prentice Hall, Englewood Cliffs, NJ, pp. 540-557.

Ruckebusch Y., Kay R. N. B., 1971. Ann. Rech. vét., 2, 99-136. 